Document downloaded from:

http://hdl.handle.net/10251/103204

This paper must be cited as:

Guirao Sánchez, AJ.; Montesinos Santalucia, V.; Zizler, V. (2018). Remarks on the set of norm-attaining functionals and differentiability. Studia Mathematica. 241(1):71-86. doi:10.4064/sm8768-6-2017

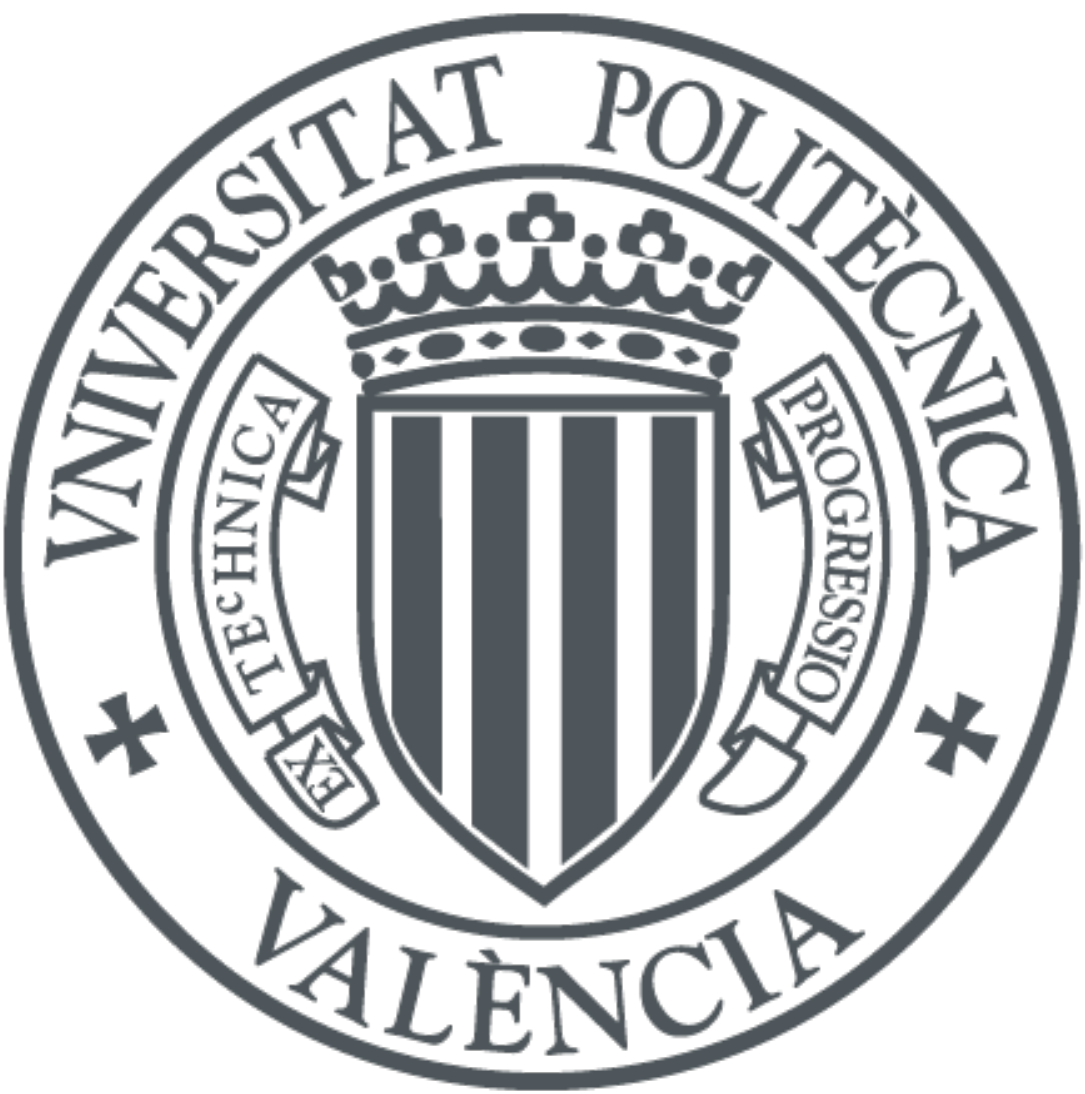

The final publication is available at

http://doi.org/10.4064/sm8768-6-2017

Copyright Institute of Mathematics, Polish Academy of Sciences

Additional Information 


\title{
REMARKS ON THE SET OF NORM-ATTAINING FUNCTIONALS AND DIFFERENTIABILITY
}

\author{
ANTONIO J. GUIRAO, VICENTE MONTESINOS, AND VACLAV ZIZLER
}

To the memory of Bob Phelps

\begin{abstract}
We use the smooth variational principle and a standard renorming to give a short direct proof to the classical Bishop-PhelpsBollobás theorem on the density of norm-attaining functionals for weakly compactly generated Banach spaces. Then we show that a slight adjustment of a known Preiss-Zajíček differentiability argument provides for a simple, useful characterization of individual norms on separable Banach spaces admitting residual sets of norm-attaining functionals in terms of Fréchet differentiability of their dual norms.
\end{abstract}

\section{INTRODUCTION}

The Bishop-Phelps theorem on the density of the norm-attaining functionals, which holds in every real Banach space, is nowadays usually proved by applying the Ekeland variational principle together with the HahnBanach separation theorem (see, e.g., [FHHMZ11, page 353]). A refinement of the theorem - approximating simultaneously functionals and "almost attaining points" by norm-attaining functionals and points of normattainment, respectively - is known as the Bishop-Phelps-Bollobás theorem (see, e.g., [Bo99, Theorem 8.11]).

In the first part of this note we show that the Bishop-Phelps-Bollobás theorem can be proved in the setting of weakly compactly generated Banach spaces by using a standard renorming theorem followed by a simple, direct application of the smooth variational principle (see, e.g., [DGZ93, Theorem 2.3], [FHHMZ11, page 355], or [Ph93, Theorem 4.10]). A separable version of a part of it appeared in [HHZ96].

Actually, the smooth variational principle provides, in spaces with a Gâteaux differentiable norm, for an effective replacement of the Ekeland variational principle. Indeed, for the application of the smooth variational

2010 Mathematics Subject Classification. Primary 46B20; Secondary 46B03, 46B26.

Key words and phrases. Smooth variational principle, Bishop-Phelps-Bollobás theorem, Gâteaux differentiable norm, norm-attaining functionals, weakly compactly generated spaces. 
principle one just needs a basic undergraduate technique (Fermat's theorem).

In the second part of this note, we use a well-known subtle PreissZajíček differentiation argument for convex functions in [PrZa84] (see also [FHHMZ11, page 414]) to characterize, in separable spaces, the norms that have residual sets of norm-attaining functionals in terms of the Fréchet differentiability of their dual norms at a dense set of points.

Since the smooth variational principle, as well as the Preiss-Zajíček argument, are built on the Baire Category theorem, most of this note is an application of this last theorem. In this connection, let us remark in passing that it is shown in [DGZ93, page 11] how also the Ekeland variational principle follows from a general variational principle proved by using the Baire Category theorem.

\section{The Bishop-Phelps-BollobÁs TheOrem VIA Differentiability}

A Banach space $X$ is said to be weakly compactly generated (WCG, in short) if there is a weakly compact set $K$ in $X$ such that $X$ is the closed linear span of $K$. The family of WCG spaces is very large: It covers all separable spaces, all reflexive spaces, $c_{0}(\Gamma)$ for all sets $\Gamma, L_{1}(\mu)$ for finite measure $\mu, C(K)$ spaces for $K$ homeomorphic to a weakly compact set in a Banach space, and more. All WCG spaces admit equivalent Gâteaux differentiable norms (cf. e.g. [FHHMZ11, page 586]). For more information on WCG spaces, see, e.g., [FHHMZ11, Chapter 13], [HMVZ08, Chapter 6], [DGZ93], and [Fa97].

If $(X,\|\cdot\|)$ is a Banach space, $X^{*}$ denotes, as usual, its dual space, i.e., the space of all continuous linear functionals on $X$ and we denote by $\|\cdot\|^{*}$ the dual norm on $X^{*}$. The closed unit ball and the unit sphere of $(X,\|\cdot\|)$ are denoted, as usual, $B_{(X,\|\cdot\|)}$ and $S_{(X,\|\cdot\|)}$, respectively (or $B_{X}$ and $S_{X}$, respectively, if the norm is understood). We say that $f \in X^{*}$ attains its norm (or that $f$ is norm-attaining) if there is $x \in S_{X}$ such that $f(x)=\|f\|^{*}$. The set of all norm-attaining functionals on $X$ is denoted by $N A(X)$ (we shall write $N A(X,\|\cdot\|)$ if we want to stress that the norm on $X$ is $\|\cdot\|$ (the set $N A(X)$ is highly sensitive to renormings of $X)$. The terms "a rotund norm" and "a strictly convex norm" are equivalent. For other nondefined concepts we refer, e.g., to [FHHMZ11].

We will first prove the Bishop-Phelps-Bollobás theorem for all spaces whose norm is Gâteaux differentiable outside the origin by using the smooth variational principle (Proposition 2.2). Observe that, in this case, 
(A) we may always define a Gâteaux differentiable bump function $b$ on $X$ such that $0 \leq b(x) \leq 1$ for all $x \in X, \operatorname{supp} b \subset B_{X}, b(0)=1$, and $\left\|b^{\prime}\right\|_{\infty}<2$.

(A bump function $b$ defined on $X$ is a real-valued function with a nonempty bounded support $\operatorname{supp} b$.)

For the reader's convenience, we reproduce here the precise statement of the smooth variational principle, as presented in [Ph93, Theorem 4.10], for the Gâteaux differentiable case:

Theorem 2.1 (Deville-Godefroy-Zizler Smooth Variational Principle ). Let $(X,\|\cdot\|)$ be a Banach space. Assume that $X$ has a Gâteaux differentiable Lipschitz bump function $b$, and that $\Phi$ is a proper lower semicontinuous bounded-below function on $X$. Then there exists a constant a $>0$ (depending only on $(X,\|\cdot\|))$ such that, for all $0<\varepsilon<1$ and for any $x_{\varepsilon} \in X$ such that $\Phi\left(x_{\varepsilon}\right)<\inf \Phi+a \varepsilon^{2}$, there exists a Gâteaux differentiable function $\varphi: X \rightarrow \mathbb{R}$ and $x_{0} \in X$ such that

(i) $\Phi+\varphi$ has a minimum at $x_{0}$.

(ii) $\|\varphi\|_{\infty}<\varepsilon$ and $\left\|\varphi^{\prime}\right\|_{\infty}<\varepsilon$.

(iii) $\left\|x_{0}-x_{\varepsilon}\right\| \leq \varepsilon$.

We may always assume that $b$ in Theorem 2.1 satisfies the conditions in (A) above. Then, from the proof of [Ph93, Theorem 4.10], the constant $a$ can be taken to be $1 /(4 M)$, where $M:=\max \left\{\left\|b^{\prime}\right\|, 1\right\}$. If $\|\cdot\|$ is Gâteaux differentiable, it is simple to produce a Gâteaux differentiable bump $b$ on $X$ that satisfies all the conditions in (A) above and, moreover, $M \leq 2$. Thus, we may take $a=1 / 8$ in Theorem 2.1.

The following is the promised proof of a particular version of the BishopPhelps-Bollobás theorem.

Proposition 2.2 (Bishop-Phelps-Bollobás). Assume that the norm $\|\cdot\|$ of a Banach space $X$ is Gâteaux differentiable. Let $f \in X^{*}$ with $\|f\|^{*} \geq 1$, $0<\varepsilon<1 / 2$, and $x_{\varepsilon} \in B_{X}$ be such that $f\left(x_{\varepsilon}\right)>\|f\|^{*}-\left(\varepsilon^{2} / 8\right)$. Then we can find a norm-attaining functional $g$ on $X$ and a point $x_{0} \in X$ such that $\|f-g\|^{*}<\varepsilon,\left\|x_{\varepsilon}-x_{0}\right\|<\varepsilon$ and $g\left(x_{0} /\left\|x_{0}\right\|\right)=\|g\|^{*}$.

Proof. First note that $\|f\|^{*} .\left\|x_{\varepsilon}\right\| \geq f\left(x_{\varepsilon}\right) \geq\|f\|^{*}-\frac{\varepsilon^{2}}{8}$. Thus

$$
\left\|x_{\varepsilon}\right\| \geq 1-\frac{\varepsilon^{2}}{8\|f\|^{*}} \geq 1-\frac{\varepsilon^{2}}{8} .
$$

Let $h: \mathbb{R} \rightarrow \mathbb{R}$ be a smooth symmetric function such that $h(0)>0$, $h(t) \geq t$ for all $t \in \mathbb{R}, h(t)=|t|$ for $|t| \geq 1, h^{\prime}(0)=0$, and $h^{\prime}(t)>0$ for $t>0$ (see Figure 1). 


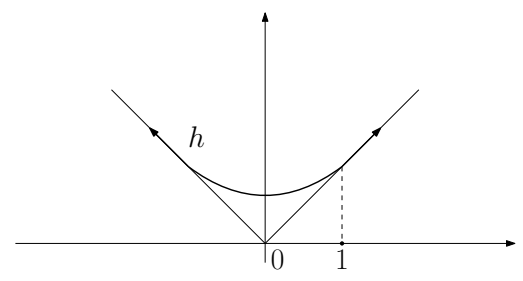

Figure 1. The function $h$

Consider the function $\Phi$ defined for $x \in X$ by

$$
\Phi(x)=\|f\|^{*} \cdot h(\|x\|)-f(x) .
$$

Then $\Phi$ is obviously continuous; it is also bounded below on $X$, due to the fact that $\Phi(x) \geq\|f\|^{*} .\|x\|-\left(\|f\|^{*} .\|x\|\right)=0$ for all $x \in X$. Moreover, $\inf _{x \in X} \Phi=0$. Indeed, if $x_{n} \in S_{X}$ satisfy $f\left(x_{n}\right) \rightarrow\|f\|^{*}$, then $\Phi\left(x_{n}\right)=\|f\|^{*}-$ $f\left(x_{n}\right) \rightarrow 0$. A direct calculation shows that $\Phi$ is Gâteaux differentiable on $X$ (use $h^{\prime}(0)=0$ ), and, if $x \in X$,

$$
\Phi^{\prime}(x)= \begin{cases}\|f\|^{*} \cdot h^{\prime}(\|x\|) \cdot\|\cdot\|^{\prime}(x)-f & \text { if } x \neq 0 \\ -f & \text { otherwise. }\end{cases}
$$

Moreover,

$$
\begin{aligned}
\Phi\left(x_{\varepsilon}\right) & =\|f\|^{*} h\left(\left\|x_{\varepsilon}\right\|\right)-f\left(x_{\varepsilon}\right) \leq\|f\|^{*} h(1)-f\left(x_{\varepsilon}\right) \\
& =\|f\|^{*}-f\left(x_{\varepsilon}\right)<\varepsilon^{2} / 8=\inf _{x \in X} \Phi+\varepsilon^{2} / 8 .
\end{aligned}
$$

By Theorem 2.1 applied to $\Phi$ and $x_{\varepsilon}$ (see (2.4)), where, as mentioned above, we take $a=1 / 8$, there is a Gâteaux differentiable function $\varphi$ on $X$ with $\|\varphi\|_{\infty}<\varepsilon$ and $\left\|\varphi^{\prime}\right\|_{\infty}<\varepsilon$ such that $(\Phi-\varphi)$ attains its minimum at some point $x_{0} \in X$ and

$$
\left\|x_{0}-x_{\varepsilon}\right\| \leq \varepsilon
$$

(so, in particular, $x_{0} \neq 0$ since $\left\|x_{0} \mid \geq\right\| x_{\varepsilon} \|-\varepsilon \geq 1-\varepsilon^{2} / 8-\varepsilon>0$, see (2.1) above). By a basic calculus, it follows that $(\Phi-\varphi)^{\prime}\left(x_{0}\right)=0$, hence

$$
\varphi^{\prime}\left(x_{0}\right)=\Phi^{\prime}\left(x_{0}\right)=\|f\|^{*} \cdot h^{\prime}\left(\left\|x_{0}\right\|\right) \cdot\|\cdot\|^{\prime}\left(x_{0}\right)-f,
$$

and, since $\left\|\varphi^{\prime}\right\|_{\infty}<\varepsilon$, we have

$$
\left\|\varphi^{\prime}\left(x_{0}\right)\right\|<\varepsilon .
$$

The functional

$$
g:=\|f\|^{*} \cdot h^{\prime}\left(\left\|x_{0}\right\|\right) \cdot\|\cdot\|^{\prime}\left(x_{0}\right)
$$

obviously attains its norm at $x:=x_{0} /\left\|x_{0}\right\|$, as the derivative of the norm at a point of the sphere is a supporting functional to the ball at this point. From (2.6) and (2.7), we deduce that $\|f-g\|^{*}<\varepsilon$. This shows the statement. 
Now we prove a renorming proposition needed. For the separable case the result appeared in [DGS-R95] and in [HHZ96].

Proposition 2.3. Let $(X,\|\cdot\|)$ be a $W C G$ Banach space. Then $X$ admits an equivalent Gâteaux differentiable norm ||$|\cdot|||$ such that

$$
N A(X,\|\cdot\|)=N A(X,\|\cdot\| \|) .
$$

Proof. By a result of D. Amir and J. Lindenstrauss (see, e.g., [FHHMZ11, Theorem 13.20]), there exists a bounded linear $w^{*}$-to- $w$-continuous one-toone operator $T$ from $X^{*}$ into some $c_{0}(\Gamma)$; this last space is considered under the (strictly convex) Day's norm $\|\cdot\|_{D}$ (see e.g. [FHHMZ11, Exercise 13.11]). Let $B_{1}$ denote the $\|\cdot\|_{D^{-}}$-losed unit ball in $c_{0}(\Gamma)$ and $B_{1}^{*}$ its corresponding dual unit ball.

The adjoint $T^{*}$ maps $c_{0}(\Gamma)^{*}$ into $X$ and the set $C:=T^{*}\left(B_{1}^{*}\right)$ is a weakly compact convex symmetric set in $X$.

Put

$$
B:=B_{(X,\|\cdot\|)}+C .
$$

Then $B$ is a closed convex symmetric set (use that $C$ is weakly compact) which is the closed unit ball of an equivalent norm $\||\cdot|||$ on $X$.

If $f \in X^{*}$ then

$$
\|f \mid\|^{*}=\sup _{B} f=\sup _{B_{(X,\|\cdot\|)}+C} f=\sup _{B_{(X,\|\cdot\|)}} f+\sup _{C} f .
$$

Since $C$ is weakly compact, it is easy to see that $f$ attains its maximum on $B$ if and only if $f$ attains its maximum on $B_{(X,\|\cdot\|)}$.

To finish the proof we shall show that $\||\cdot|\|^{*}$ is strictly convex. Then, by the Šmulyan test (see, e.g., [FHHMZ11, page 344]), the norm || · ||| will be Gâteaux differentiable.

To show the strict convexity of \|\|$\cdot \|^{*}$ calculate, for $f \in X^{*}$,

$$
\begin{aligned}
\|f\|^{*} & =\sup _{B_{(X,\|\cdot\|)}+C} f=\sup _{B_{(X,\|\cdot\|)} f+\sup _{C} f} \\
& =\|f\|^{*}+\sup _{T^{*}\left(B_{1}^{*}\right)} f=\|f\|^{*}+\sup _{B_{1}^{*}}(T f)=\|f\|^{*}+\|T f\|_{D} .
\end{aligned}
$$

Since the norm $\|\cdot\|_{D}$ is strictly convex, it is standard to show that $\|\cdot\| \|^{*}$ is strictly convex, too (see, e.g., [FHHMZ11, page 335]). Indeed, assume that $\||f+g|\|^{*}=\|\| f\left|\left\|^{*}+\right\|\right|\|g \mid\|^{*}$ and $g \neq 0$. Then a similar equality holds for both of the summands. Thus, because of the convexity, $\|T f+T g\|_{D}=$ $\|T f\|_{D}+\|T g\|_{D}$ and $T g \neq 0$. Due to the strict convexity of Day's norm, we have $T f=\lambda T g$ for some $\lambda>0$. Thus $f=\lambda g$ as $T$ is one-to-one. This shows that ||$\cdot \mid \|^{*}$ is strictly convex. 
From Propositions 2.2 and 2.3, we directly get the following Corollary 2.4 below (a special case of the general Bishop-Phelps-Bollobás theorem).

Corollary 2.4 (Bishop-Phelps-Bollobás, WCG case). Let $(X,\|\cdot\|)$ be a $W C G$ Banach space. Then, given $0<\varepsilon<1 / 2, f \in S_{X^{*}}$ and $x_{\varepsilon} \in S_{X}$ such that $f\left(x_{\varepsilon}\right)>1-\varepsilon^{2} / 128$, there exists $g \in X^{*}$ and $x \in S_{X}$ with $\|f-g\|^{*}<\varepsilon$, $\left\|x_{\varepsilon}-x\right\|<\varepsilon$ and $g(x)=\|g\|^{*}$.

Proof. Fix $0<\varepsilon<1 / 2, f \in S_{X^{*}}$ and $x_{\varepsilon} \in S_{X}$ as in the statement. Fix $\delta:=\delta(\varepsilon)>0$ (to be defined later). By letting $\|T\|<\delta$ in the proof of Proposition 2.3 (where $T:\left(X^{*},\|\cdot\|^{*}\right) \rightarrow\left(c_{0}(\Gamma),\|\cdot\|_{D}\right)$ is the continuous linear mapping whose existence is guaranteed by the WCG character of $X$ ), we may find a Gâteaux differentiable norm |||||| such that

$$
\|\| x\|\mid \leq\| x\|\leq(1+\delta)\|\|x\| \|, \text { for all } x \in X
$$

(and so

$$
\left.\|h\|^{*} \leq\|h\|^{*} \leq(1+\delta)\|h\|^{*} \text { for all } h \in X^{*}\right) .
$$

Observe that $\|y\|<\delta$ for every $y \in C\left(:=T^{*}\left(B_{1}^{*}\right)\right.$, where $B_{1}^{*}$ is the closed unit ball of $\left.\left(\ell_{1}(\Gamma),\|\cdot\|_{D}^{*}\right)\right)$. Let $y_{\varepsilon} \in C$ be the point where $f$ attains its maximum on $C$, so that $\|f \mid\|^{*}=\|f\|^{*}+f\left(y_{\varepsilon}\right)$ (see (2.11) above). Set $z_{\varepsilon}=$ $x_{\varepsilon}+y_{\varepsilon}\left(\right.$ so $z_{\varepsilon} \in B_{\|\cdot\| \cdot \|}$, see (2.9) above). Obviously $f\left(z_{\varepsilon}\right)>\|\| f \|^{*}-\varepsilon^{2} / 128$. Put $r:=\varepsilon / 4$, so $f\left(z_{\varepsilon}\right)>\|\| f \|^{*}-r^{2} / 8$. It follows from Proposition 2.2 that there exists $g \in X^{*}$ and $z \in X$ such that \|\|$f-g\left\|^{*}<r,\right\|\left\|z_{\varepsilon}-z\right\| \|<r$ and $g\left(z /|\|z \mid\|)=\mid\|g\|^{*}\right.$. In particular,

$$
\|f-g\|^{*} \leq\|\| f-g \|^{*}<r \quad(<\varepsilon) .
$$

Put $z /\|z\|=x+y$, where $\|x\| \leq 1$ and $y \in C$ (see again (2.9) above). Clearly, $g$ attains its $\|\cdot\|^{*}$-norm at $x$, so $\|x\|=1$.

Then we have

$$
\begin{aligned}
x_{\varepsilon}-x & =z_{\varepsilon}-y_{\varepsilon}-x \\
& =z_{\varepsilon}-z+z\left(1-\frac{1}{\|z \mid\|}\right)-y_{\varepsilon}+y,
\end{aligned}
$$

hence

$$
\left\|x_{\varepsilon}-x\right\| \leq\left\|z_{\varepsilon}-z\right\|+\|z\| .\left|1-\frac{1}{\|z \mid\|}\right|+\left\|y_{\varepsilon}\right\|+\|y\| .
$$

From (2.12) it is not difficult to show that, by choosing $0<\delta$ small enough, we have $\left\|x_{\varepsilon}-x\right\|<\varepsilon$. Let us provide the details for the sake of completeness. For this, let us estimate the terms in (2.12): First we have

$$
\left\|z-z_{\varepsilon}\right\| \leq(1+\delta)\left\|z-z_{\varepsilon}\right\| \mid<(1+\delta) r .
$$


We collect now some inequalities needed:

$$
\begin{array}{r}
\left\|z_{\varepsilon}\right\| \leq\left\|x_{\varepsilon}\right\|+\left\|y_{\varepsilon}\right\|<1+\delta, \\
\|z\| \leq\left\|z_{\varepsilon}\right\|+\left\|z-z_{\varepsilon}\right\|<(1+\delta)(1+r), \\
\left\|z_{\varepsilon} \mid\right\| \leq\left\|z_{\varepsilon}\right\|<1+\delta, \\
\left\|z \left|\|\leq\| z-z_{\varepsilon}\left\|\left|+\left\|z_{\varepsilon} \mid\right\|<r+(1+\delta),\right.\right.\right.\right. \\
1-\delta<\left\|x_{\varepsilon}\right\|-\left\|y_{\varepsilon}\right\| \leq\left\|z_{\varepsilon}\right\|, \\
\frac{1-\delta}{1+\delta}<\left\|z_{\varepsilon}\right\|, \\
\frac{1-\delta}{1+\delta}-r<\left\|z_{\varepsilon}\right\|-\left\|z-z_{\varepsilon}\right\| \leq\|\| z \| .
\end{array}
$$

Observe that $(1-r) /(1+r)>0$. By choosing $0<\delta<(1-r) /(1+r)$ we can guarantee that $[(1-\delta) /(1+\delta)]-r>0$. Thus, by using $(2.17)$ and $(2.20)$ above,

$$
\frac{-2 \delta-r(1+\delta)}{(1-\delta)-r(1+\delta)}<1-\frac{1}{\|z\|}<\frac{r+\delta}{r+1+\delta}
$$

It is simple to prove that

SO

$$
\frac{2 \delta+r(1+\delta)}{(1-\delta)-r(1+\delta)}>\frac{r+\delta}{r+1+\delta}
$$

$$
\left|1-\frac{1}{\|\| z \mid \|}\right|<\frac{2 \delta+r(1+\delta)}{(1-\delta)-r(1+\delta)} .
$$

Note that $0<r<\varepsilon<1 / 2$, so we may take $\delta>0$ small enough so that $\delta+r+r \delta<1 / 2$. Then, $(1-\delta)-r(1+\delta)>1 / 2$, and from (2.22) we get

$$
\left|1-\frac{1}{|\|z \mid\|}\right|<2(2 \delta+r(1+\delta)) \text {. }
$$

Finally, we have, from (2.12), and by using (2.13), (2.15), and (2.23) in this order,

$$
\left\|x_{\varepsilon}-x\right\|<(1+\delta) r+(1+\delta)(1+r) 2(2 \delta+r+r \delta)+2 \delta,
$$

and it is easy to show that, for $\delta>0$ small enough (and satisfying the conditions encountered above),

$$
(1+\delta) r+(1+\delta)(1+r) 2(2 \delta+r+r \delta)+2 \delta<4 r(=\varepsilon),
$$

since this is equivalent to

$$
\delta\left(9 r+6+4 r^{2}+4 \delta+6 r \delta+2 r^{2} \delta\right)<r-2 r^{2}
$$

(note that $r-2 r^{2}>0$ ). This finalizes the proof.

\section{Remark 2.5.}


(1) Observe that Corollary 2.4 is in fact a constructive version - for the WCG case - of the Bishop-Phelps-Bollobás theorem, which is usually proved by non-constructive techniques. Indeed, (2.8) in the proof of Proposition 2.2 gives the formula of the functional that attains the norm in the Gâteaux case, and (2.10) in the proof of Proposition 2.3 gives the formula for the equivalent strictly convex dual norm — and so for the sought equivalent Gâteaux norm on the space-. It gives no information about the point $x$ though.

(2) The reader would have no difficulty to simplify the method of proof of Proposition 2.3 for separable spaces, where injections into $\ell_{2}$ can be used.

(3) In proving just the Bishop-Phelps theorem, the situation is easier, and for the reader's convenience we include here the needed adjustment in detail.

Theorem (Bishop-Phelps, Gâteaux differentiability case) Assume that the norm $\|\cdot\|$ of a Banach space $X$ is Gâteaux differentiable. Then $N A(X,\|\cdot\|)$ is norm-dense in $X^{*}$.

Proof. Given $f \in X^{*}$ (without loss of generality we may assume that $\|f\|^{*}=1$ ), and $1>\varepsilon>0$, we need to show that there is $g \in X^{*}$ that attains its norm and $\|f-g\|^{*}<\varepsilon$. To this end, consider the function $\Phi$ defined for $x \in X$ by

$$
\Phi(x)=\|x\|^{2}-f(x) .
$$

Then $\Phi$ is continuous and bounded below on $X$. Indeed,

$$
\Phi(x) \geq\|x\|^{2}-\|f\|^{*} \cdot\|x\| \geq-\frac{\left(\|f\|^{*}\right)^{2}}{4} \text { for all } x \in X .
$$

Moreover, $\Phi$ is Gâteaux differentiable, and

$$
\Phi^{\prime}(x)= \begin{cases}2\|x\| \cdot\|\cdot\|^{\prime}(x)-f, & \text { if } x \in X, x \neq 0, \\ -f & \text { otherwise. }\end{cases}
$$

Thus, by the smooth variational principle, there is a Lipschitz and Gâteaux differentiable function $\varphi$ on $X$ with $\|\varphi\|_{\infty}<\varepsilon$ and $\left\|\varphi^{\prime}\right\|_{\infty}<$ $\varepsilon$ such that $(\Phi-\varphi)$ attains its minimum at some point $x_{0} \in X$. Therefore, $(\Phi-\varphi)^{\prime}\left(x_{0}\right)=0$.

If $x_{0}=0$, we get $\varphi^{\prime}(0)=\Phi^{\prime}(0)=-f$, a contradiction with the fact that $\|f\|^{*}=1$ and $\left\|\varphi^{\prime}(0)\right\|<\varepsilon<1$. We have then $x_{0} \neq 0$. Therefore

$$
\varphi^{\prime}\left(x_{0}\right)=\Phi^{\prime}\left(x_{0}\right)=2\left\|x_{0}\right\| \cdot\|\cdot\|^{\prime}\left(x_{0}\right)-f
$$


moreover, $\left\|\varphi^{\prime}\left(x_{0}\right)\right\|<\varepsilon$. The functional $2\left\|x_{0}\right\| \cdot\|\cdot\|^{\prime}\left(x_{0}\right)$ obviously attains its norm, and we can thus put

$$
g:=2\left\|x_{0}\right\| \cdot\|\cdot\|^{\prime}\left(x_{0}\right) .
$$

(4) Note that Proposition 2.3 does not hold in general if "Gâteaux differentiability" there is replaced by "Fréchet differentiability", even in spaces whose duals are separable. Indeed, M. D. Acosta and M. Ruiz Galán proved in [AcR-G98] that every Banach space can be renormed so that the new set $N A(X)$ has an interior point. On the other hand they proved in the same paper that $N A(X)$ has empty interior whenever the norm of a nonreflexive separable space $X$ is Fréchet differentiable.

\section{The Residuality of THE SET OF NORM-ATtAining FUnCTIONALS}

\section{AND DIFFERENTIABILITY}

A norm is said to be dentable if its unit ball is dentable, i.e. contains arbitrarily small-diameter cuts by open halfspaces, cuts called slices. This is equivalent to say that given $\varepsilon>0$ there exists $x_{0} \in B_{X}$ such that $x_{0} \notin$ $\overline{\mathrm{co}}\left(B_{X} \backslash\left\{x \in X:\left\|x-x_{0}\right\|<\varepsilon\right\}\right)$.

It is well known (see, e.g., Theorem 3.4 below) that if $X$ is a Banach space then all equivalent norms on $X$ are dentable if and only if for every equivalent norm the set $N A(X)$ is residual, and this happens if, and only if, $X$ has the Radon-Nikodým property. In contrast, for the Day norm $\|\cdot\|_{D}$ on $c_{0}$ the set $N A\left(c_{0},\|\cdot\|_{D}\right)$ is even dense $G_{\delta}$ (see Remark 3.3.2 below), while the set $N A\left(c_{0},\|\cdot\|_{\infty}\right)$ consists exactly of the finitely supported vectors (see e.g. [FHHMZ11, page 142]), so it is of first category (cf., e.g., [FHHMZ11, page 173]). So the following proposition comes handy in general separable spaces. It is of good practical use since, usually, it is much easier to decide if the dual norm is Fréchet differentiable at a dense set of points of the dual space than to decide on the residuality of the set of norm-attaining functionals. The proof is a slight adjustment of a simple, clever argument of D. Preiss and L. Zajíček in [PrZa84] (see also [FHHMZ11, page 414]) which is a delightful combination of convexity and Baire category arguments.

Proposition 3.1 (D. Preiss and L. Zajíček, norm-attaining version). Let $(X,\|\cdot\|)$ be a separable Banach space. Then the set $N A(X,\|\cdot\|)$ is residual in $\left(X^{*},\|\cdot\|^{*}\right)$ if and only if $\|\cdot\|^{*}$ is Fréchet differentiable at points of a dense subset of $\left(X^{*},\|\cdot\|^{*}\right)$. 
Proof. 1) To prove the necessity, assume that $N A(X,\|\cdot\|)$ is residual. We adjust the Preiss-Zajíček argument in [PrZa84] to show that $\|\cdot\|^{*}$ is Fréchet differentiable at a dense set of points in the dual, which is automatically $G_{\delta}$ (see [FHHMZ11, page 357]). Let $P$ be a $G_{\delta}$ dense set in $\left(X^{*},\|\cdot\|^{*}\right)$ included in $N A(X,\|\cdot\|)$ (see, e.g., [MZZ15, Theorem 646]). The space $\left(P,\|\cdot\|^{*}\right)$ is a Baire space (see, e.g., the proof of [MZZ15, Theorem 589]).

Let $A$ be the set of all points in $P$ where $\|\cdot\|^{*}$ is not Fréchet differentiable. We shall show that $A$ is of first category in $P$. This will finish the proof of this implication.

For every $f \in A$ we find $x_{f} \in S_{X}$ where $f$ attains its norm. Then $\|f+h\|^{*}-\|f\|^{*} \geq h\left(x_{f}\right)$ for every $h \in X^{*}$. Since $\|\cdot\|^{*}$ is not Fréchet differentiable at points of $A$, we may find, by the Šmulyan's test, $m_{f} \in \mathbb{N}$ such that

$$
\limsup _{h \rightarrow 0} \frac{\|f+h\|^{*}-\|f\|^{*}-h\left(x_{f}\right)}{\|h\|^{*}}>\frac{1}{m_{f}} .
$$

For $m \in \mathbb{N}$ put $A_{m}:=\left\{f \in A: \quad m_{f}=m\right\}$ and consider a cover of $B_{X}$ by open balls in $X$ of radius $1 /(12 m)$. Since $X$ is separable, by the Lindelöf property we can find a countable subfamily $\left\{B_{k}^{m}\right\}_{k}$ of these balls that covers $B_{X}$. For $k \in \mathbb{N}$ define $A_{m, k}=\left\{f \in A_{m}: x_{f} \in B_{k}^{m}\right\}$. Observe that, for $f, g \in A_{m k}$, we have $\left\|x_{f}-x_{g}\right\|<1 /(6 m)$. From the Šmulyan's test we have $A=\bigcup_{m, k} A_{m, k}$. Hence it is enough to show that $A_{m, k}$ is nowhere dense in $P$ for each $m, k$.

The rest of the argument refers to the topological space $\left(P,\|\cdot\|^{*}\right)$. Assume, on the contrary, that there exists a nonempty open subset $O$ of $\overline{A_{m, k}}$. Find $f \in O \cap A_{m, k}$ and $r>0$ such that $B(f, r) \subset O$. Due to the fact that $f \in A_{m}$ we may find $h \in X^{*}$ such that $\|h\|^{*}<r / 2$ and

$$
\|f+h\|^{*}-\|f\|^{*}>\frac{\|h\|^{*}}{m}+h\left(x_{f}\right) .
$$

Since $P$ is $\|\cdot\|^{*}$-dense in $X^{*}$, we may assume, without loss of generality, that $f+h \in P$.

We claim that $B\left(f+h, \frac{\|h\|^{*}}{3 m}\right) \cap A_{m, k}=\emptyset$. Since $B\left(f+h, \frac{\|h\|^{*}}{3 m}\right) \subset B(f, r) \subset$ $O \subset \overline{A_{m, k}}$, we shall get a contradiction.

In order to prove the claim, assume, on the contrary, that there is $g \in$ $B\left(f+h, \frac{\|h\|^{*}}{3 m}\right) \cap A_{m, k}$. In particular

$$
\|f+h-g\|^{*}<\frac{\|h\|^{*}}{3 m}
$$

and so

$$
\|f-g\|^{*}<\frac{\|h\|^{*}}{3 m}+\|h\|^{*}<2\|h\|^{*}
$$




$$
\|f+h\|^{*}-\|g\|^{*} \leq\|f+h-g\|^{*}<\frac{\|h\|^{*}}{3 m},
$$

and, by the fact that $f, g \in A_{m, k}$,

$$
\left\|x_{f}-x_{g}\right\|<\frac{1}{6 m} .
$$

Due to the definition of $x_{g}$ we have

$$
\|f\|^{*}-\|g\|^{*}=\|g+(f-g)\|^{*}-\|g\|^{*} \geq(f-g)\left(x_{g}\right) .
$$

By using successively (3.2), (3.7), (3.3), (3.4), and (3.6), in this order, we get

$$
\begin{aligned}
\|f+h\|^{*}-\|g\|^{*} & =\|f+h\|^{*}-\|f\|^{*}+\|f\|^{*}-\|g\|^{*} \\
& >\frac{\|h\|^{*}}{m}+h\left(x_{f}\right)+(f-g)\left(x_{g}\right) \\
& =\frac{\|h\|^{*}}{m}+(f+h-g)\left(x_{f}\right)-f\left(x_{f}\right)+g\left(x_{f}\right)+(f-g)\left(x_{g}\right) \\
& =\frac{\|h\|^{*}}{m}+(f+h-g)\left(x_{f}\right)+(f-g)\left(x_{g}-x_{f}\right) \\
& >\frac{\|h\|^{*}}{m}-\frac{\|h\|^{*}}{3 m}-\frac{\|h\|^{*}}{3 m}=\frac{\|h\|^{*}}{3 m},
\end{aligned}
$$

and this is in contradiction with (3.5). This proves the claim, and so ends the proof of the necessity implication.

2) To prove the sufficiency, assume now that the dual norm is differentiable at a dense set of points in $\left(X^{*},\|\cdot\|^{*}\right)$. Note that the set of Fréchet differentiability is always $G_{\delta}$ (see, e.g., [FHHMZ11, page 357]), and that if the norm is Fréchet differentiable at the point $f$, then the Šmulyan's test (cf., e.g., [FHHMZ11, page 343]) implies that $f$ attains its norm. So, the set of norm-attaining functionals contains a dense $G_{\delta}$ set, hence it is residual in $X^{*}$.

Recall that $x \in S_{X}$ is a strongly exposed point of $B_{X}$ if there is $f \in S_{X^{*}}$ such that $f(x)=1$ and $\left\|x_{n}-x\right\| \rightarrow 0$ whenever $x_{n} \in B_{X}$ are such that $f\left(x_{n}\right) \rightarrow 1$. We say then that $f$ strongly exposes $x$, or that $x$ is strongly exposed by $f$. From the Šmulyan test it follows that there is a duality between strongly exposed points of $B_{X}$ and points of Fréchet differentiability of the dual norm. Namely, if $x \in S_{X}$ and $f \in S_{X^{*}}$ are such that $f(x)=1$ and $f$ is a point of Fréchet differentiability of the dual norm then $x$ is strongly exposed by $f$.

From this duality and from Proposition 3.1 we get the following corollary by a standard separation argument. 
Corollary 3.2. Let $(X,\|\cdot\|)$ be a separable Banach space. If $N A(X,\|\cdot\|)$ is residual in $X^{*}$, then $B_{(X,\|\cdot\|)}$ is the closed convex hull of its strongly exposed points.

\section{Remark 3.3.}

(1) Proposition 3.1 and Corollary 3.2 above should be compared with the following J. Bourgain-Ch. Stegall result, see [Bour83, Theorem 3.5.5]: If $(X,\|\cdot\|)$ is a separable Banach space and $N A(X,\|\cdot\|)$ is of second category in $\left(X^{*},\|\cdot\|^{*}\right)$, then $B_{X}$ is dentable.

Note that in Corollary 3.2 we assume more than in the BourgainStegall result and get more, precisely that $B_{X}$ is the closed convex hull of its strongly exposed points.

At the end of this note we shall review some known results concerning these properties.

(2) Unlike the Gâteaux smoothness in WCG spaces, the rotundity of the norm contributes to the quality of $N A(X)$ for this norm. The most transparent situation is the case of the LUR norms (the norm $\|\cdot\|$ on a Banach space is said to be locally uniformly rotund (LUR, in short) if $\left\|x_{n}-x\right\| \rightarrow 0$ whenever $x_{n}, x \in S_{X}$ are such that $\left\|x_{n}+x\right\| \rightarrow 2$ ):

If $\|\cdot\|$ on $X$ is LUR, a functional attains its norm if and only if it is a point of Fréchet differentiability of $\|\cdot\|^{*}$. Indeed, if it is a point of Fréchet differentiability of $\|\cdot\|^{*}$, then it attains its norm by the Šmulyan test, as we mentioned in the proof of Proposition 3.1, sufficiency part. If in turn the functional attains its norm, then, due to the LUR property of $\|\cdot\|$, it is a point of Fréchet differentiability of $\|\cdot\|^{*}$, again by the Šmulyan test.

Thus the set of functionals that attain their norm coincides in this case with the set of points of Fréchet differentiability of $\|\cdot\|^{*}$, so this is a dense set in $\left(X^{*},\|\cdot\|^{*}\right)$ by the Bishop-Phelps theorem, as well as it is automatically $G_{\delta}$ (cf., e.g., [FHHMZ11, page 357]). Summarizing, if the norm $\|\cdot\|$ on a Banach space $X$ is LUR, then $N A(X,\|\cdot\|)$ is a dense $G_{\delta}$ subset of $\left(X^{*},\|\cdot\|^{*}\right)$.

Since S. Troyanski proved that every WCG space admits an equivalent LUR norm (see, e.g., [FHHMZ11, page 587]), we have that every WCG space $(X,\|\cdot\|)$ has an equivalent norm $|\|\cdot \mid\|$ so that $N A\left(X,|\|\cdot \mid\|)\right.$ is dense $G_{\delta}$-so residual - in $X^{*}$.

These facts should be compared with Proposition 3.1 and Corollary 3.2. 
(3) In the paragraph before Proposition 3.1 we mentioned that $N A\left(c_{0}, \| \cdot\right.$ $\|_{\infty}$ ) is of first category. It cannot be $G_{\delta}$ by that proposition (it is always $\|\cdot\|_{\infty}^{*}$-dense), as the dual norm on $\ell_{1}$ is nowhere Fréchet differentiable.

(4) Note that $N A(X,\|\cdot\|)$ can be of first category for a norm $\|\cdot\|$ with dentable ball (R. Bourgin [Bour83, Example 3.5.8]).

(5) If $X$ is separable and non-reflexive, then $N A(X)$ is not $G_{\delta}$ in $\left(X^{*}, w^{*}\right)$ ([DGS-R95]).

(6) R. Kaufman proved in [Ka91] that for separable Banach spaces $X$, $N A(X)$ is an analytic set in $\left(X^{*}, w^{*}\right)$.

Also, R. Kaufman showed in [Ka91] that every separable nonreflexive Banach space $X$ can be renormed so that $N A(X)$ is not Borel in $\left(X^{*},\|\cdot\|^{*}\right)$. He proved in $[\mathrm{Ka} 00]$ that a nonreflexive space with separable dual can be renormed by a Fréchet differentiable norm so that $N A(X)$ is not Borel (see also [Ku11]). On the other hand, M. D. Acosta and the second named author of this note proved that every separable non-reflexive Banach space $X$ can be renormed so that the set of norm-attaining functionals has empty interior in $\left(X^{*},\|\cdot\|^{*}\right)$ ([AcMo07]).

Thus it follows that the behaviour of $N A(X)$ is highly sensitive to renormings: For example, we mentioned in Remark 3.3.3 above that $N A\left(c_{0},\|\cdot\|_{\infty}\right)$ is of first category (and dense not $G_{\delta}$ ), while if $\|\cdot \cdot\|$ is a Fréchet differentiable LUR norm on $c_{0}$ (see, e.g., [FHHMZ11, page 387$]), N A\left(c_{0},|\|\cdot \mid\|)\right.$ is dense $G_{\delta}$ in $\left(X^{*},\|\cdot\|^{*}\right)$, and it has no interior point (see Remark 2.5.4).

(7) R. Kaufman showed in [Ka91] that $N A(X)$ is Borel (even in the $w^{*}$ topology) for separable rotund norms. Later on, O. Kurka showed in $[\mathrm{Ku} 11]$ that every separable nonreflexive space can be renormed so that the Borel class of $N A(X)$ is arbitrarily high.

(8) Overall, the best behavior of the sets of norm-attaining functionals for all equivalent norms on the space are met in the class of Banach spaces having the Radon-Nikodým property (RNP, in short: A Banach space $X$ has the RNP property if and only if any bounded set in it is dentable).

Below, we indicate the sources of proofs to the following theorem, which is a result of the work of many mathematicians. For simplicity, we restrict ourselves here to separable spaces. 
Theorem 3.4. Let $X$ be a separable Banach space. Then the following are equivalent.

(a) $X$ has the RNP property.

(b) The ball of every equivalent norm on $X$ is dentable.

(c) The ball of every equivalent Gâteaux differentiable norm on $X$ is dentable.

(d) The dual norm of every equivalent norm on $X$ is Fréchet differentiable at a dense set of points in $X^{*}$.

(e) For every equivalent norm on $X$, the set $N A(X)$ is residual in $X^{*}$.

(f) For every equivalent norm on $X$, the set $N A(X)$ is of second category in $X^{*}$.

Sources of possible proofs. $(a) \Leftrightarrow(b)$ [Di75, page 219].

$(b) \Rightarrow(c)$ is trivial.

$(c) \Rightarrow(b)$ : Arguing by contradiction, assume that $\|\cdot\|_{1}$ is a norm on $X$ such that its closed unit ball $B_{1}$ is not dentable. Since $X$ is separable, there exists an equivalent norm $\|\cdot\|_{2}$ on $X$, with closed unit ball $B_{2}$, whose dual norm is strictly convex (see the proof of Proposition 2.3 above). As we saw there, $\|\cdot\|_{1}^{*}+\|\cdot\|_{2}^{*}$ is strictly convex, so its predual norm $\|\cdot\|$ is Gâteaux differentiable. Its closed unit ball $B$ is the closure of the set $B_{1}+B_{2}$. To show that $B$ is not a dentable set, it is enough to show that the set $B_{1}+B_{2}$ is not dentable. Since $B_{1}$ is not dentable, let $\varepsilon>0$ be such that every slice of $B_{1}$ has diameter greater than or equal to $\varepsilon$. Let $b_{1} \in B_{1}$ and $b_{2} \in B_{2}$ and an open halfspace $H$ be such that $b_{1}+b_{2} \in H$. Consider the halfspace $H-b_{2}$. Then $b_{1} \in H-b_{2}$ and thus there are points $b_{1}^{\prime}$ and $b_{1}^{\prime \prime}$ in $\left(H-b_{2}\right) \cap B_{1}$ whose distance from each other is greater than or equal to $\varepsilon$. Then $b_{1}^{\prime}+b_{2}$ and $b_{1}^{\prime \prime}+b_{2}$ are at distance greater than or equal to $\varepsilon$, and both are in $\left(B_{1}+B_{2}\right) \cap H$.
$(a) \Leftrightarrow(d)[\mathrm{Co} 76]$.
$(e) \Leftrightarrow(d)$ Proposition 3.1.
$(f) \Rightarrow(b)$ follows from the Bourgain-Stegall result mentioned in Remark 3.3.1 above.

$$
(e) \Rightarrow(f) \text { is obvious. }
$$

(9) M. Edelstein constructed in [Ede73] an equivalent dentable norm on $c_{0}$ that has no extreme point in the unit sphere. In the same paper, he also constructed an equivalent strictly convex norm ||$\cdot|| \mid$ on $c_{0}$ whose unit ball is not dentable. By the Bourgain-Stegall result 
mentioned in Remark 3.3.1 above, $N A\left(c_{0},\||\cdot|\|\right)$ is of first category. This should be compared with the results of R. Kaufman mentioned in Remarks 3.3.6 and 3.3.7 above.

Actually there are many norms like this. First a definition: A Banach space $X$ is said to have the $C P C P$ property if every closed bounded convex set in $X$ contains a point where the norm topology and the weak topologies coincide. From Theorem 3.4 and Proposition 3.5 below, every separable Banach space with the RNP property has the CPCP property. The predual of the James tree space is an example of a space with the CPCP property that does not have the RNP property (see, e.g. [DGHZ87]). For more on spaces with the CPCP property we refer, e.g., to [DGHZ87], [DGZ93], [EdWh84], and references therein.

Proposition 3.5. Let $X$ be a separable Banach space. Then the following are equivalent:

(a) $X$ has the CPCP property.

(b) Every equivalent strictly convex norm on $X$ is dentable.

(c) For every equivalent strictly convex norm on $X, N A(X)$ is residual.

(d) For every equivalent strictly convex norm on $X, N A(X)$ is of second category.

Proof. (a) $\Leftrightarrow($ b) is in [DGHZ87, Corollary 6].

It is proved in [DGHZ87, Theorem 1] that a separable Banach space has the CPCP property if and only if the dual of every equivalent strictly convex norm is Fréchet differentiable at a dense set of points, which by Proposition 3.1 gives $(\mathrm{a}) \Leftrightarrow(\mathrm{c})$.

Obviously, if (c) holds, then (d) holds.

If (d) holds, then by the Bourgain-Stegall result mentioned in Remark 3.3.1 above, the unit ball of every equivalent strictly convex norm is dentable. This is (b).

(10) If $X^{*}$ is separable we can say more (Proposition 3.6 below). For the proof of it we need to recall a definition: A norm $\|\cdot\|$ of a Banach space $X$ is said to be weakly uniformly rotund (WUR, in short) if $x_{n}-y_{n} \rightarrow 0$ in the weak topology of $X$ whenever $x_{n}, y_{n} \in S_{X}$ are such that $\left\|x_{n}+y_{n}\right\| \rightarrow 2$. If $X$ is a dual space and the weak topology is replaced with the weak* topology, we call the dual norm weak* uniformly rotund ( $\mathrm{W}^{*} \mathrm{UR}$, in short). Each of the concepts WUR and 
$\mathrm{W}^{*} \mathrm{UR}$ obviously imply strict convexity. We need to know that a norm on a Banach space is uniformly Gâteaux differentiable if, and only if, the dual norm is $\mathrm{W}^{*} \mathrm{UR}$, and that the norm is WUR if, and only if, the dual norm is uniformly Gâteaux differentiable (see, e.g., [DGZ93, Theorem 6.7]).

Proposition 3.6. Let $X$ be a Banach space such that $X^{*}$ is separable. Then the following are equivalent:

(a) $X$ has the CPCP property.

(b) Every equivalent WUR norm on $X$ is dentable.

(c) Every equivalent norm on $X$ that is both WUR and uniformly Gâteaux differentiable, is dentable.

(d) For every equivalent norm on $X$ that is both WUR and uniformly Gâteaux differentiable, the set $N A(X)$ is residual.

(e) For every equivalent norm on $X$ that is both WUR and uniformly Gâteaux differentiable, the set $N A(X)$ is of second category.

Proof. From a result in [DGHZ87] (see also [HMZ11, Theorem 40]) we see that $(\mathrm{a}) \Leftrightarrow(\mathrm{b})$. So, if $X$ does not have the CPCP property, then there is a WUR norm $\|\cdot\|_{1}$ on $X$ that is not dentable. Since $X^{*}$ is separable, there is a norm $\|\cdot\|_{2}$ that is WUR and its dual norm is $W^{*} \mathrm{UR}$ (see, e.g., [DGZ93, Theorem 6.7 (ii)] and [DGZ93, Theorem 7.1 (ii)]). The sum of the dual norms $\|\cdot\|_{3}^{*}:=\|\cdot\|_{1}^{*}+\|\cdot\|_{2}^{*}$ is $\mathrm{W}^{*} \mathrm{UR}$ because $\|\cdot\|_{2}^{*}$ is. Moreover $\|\cdot\|_{3}^{*}$ is uniformly Gâteaux differentiable as both norms are uniformly Gâteaux differentiable. So its predual norm $\|\cdot\|_{3}$ is WUR and uniformly Gâteaux differentiable. As in the proof of Theorem 3.4, we see that $\|\cdot\|_{3}$ is not dentable. Thus non (a) implies non (c). So, (c) implies (a). Obviously, (b) $\Rightarrow$ (c). Thus (a), (b) and (c) are equivalent. The implication $(\mathrm{a}) \Rightarrow(\mathrm{b})$ in Proposition 3.5 above shows that $(\mathrm{a}) \Rightarrow(\mathrm{d})$ here. Obviously, $(\mathrm{d}) \Rightarrow(\mathrm{e})$. From the Bourgain-Stegall result in Remark 3.3.1, (e) $\Rightarrow(\mathrm{c})$.

Observe that (c), (d), and (e) in Proposition 3.6 can be formulated for equivalent WUR and Gâteaux differentiable norms instead. This gives extra information if starting from the CPCP property, while the version with WUR and uniformly Gâteaux differentiable norms demands less requirements for getting the CPCP property.

Problem 3.7. Is Proposition 3.1 true if we assume instead of the separability of $X$ that $X$ admits an equivalent LUR norm? 
Acknowledgements. The third named author would like to express the gratitude of the whole Prague Banach space community to the late Bob Phelps for his constant long time moral support and participation in the annual international Prague Winter Schools for young researchers in Banach spaces. This research was partly supported by MICINN and FEDER Projects MTM2014-57838-C2-1-P, MTM2014-57838-C2-2-P. The first named author was also partly supported by Fundación Séneca, Región de Murcia grant 19368/PI/14.

The authors want to thank the referee for the careful reading of the manuscript and the invaluable observations that helped to improve the final version.

\section{REFERENCES}

[AcR-G98] M. D. Acosta and M. Ruiz Galán, New characterizations of the reflexivity in terms of the set of norm-attaining functionals, Canadian Math. Bull. 41 (3), 1998, 279-289.

[AcMo07] M. D. Acosta and V. Montesinos, On a problem of Namioka on norm-attaining functionals, Math. Z. 256 (2007), 295-300.

[BiPh61] E. Bishop and R. R. Phelps, A proof that every Banach space is subreflexive, Bull. Amer. Math. Soc. 67 (1961), 877-898.

[Bo99] B. Bollobás, Linear Analysis, 2nd Ed., Cambridge Math. Textbooks, Cambridge University Press, 1999.

[Bou79] J. Bourgain, La propriété de Radon-Nikodým, Publications de l'Université Pierre et Marie Curie, 36 (1979).

[Bour83] R. Bourgin, Geometric aspects of convex sets with the RadonNikodým property, Lecure Notes in Mathematics, 93, Springer, 1983.

[Co76] J. B. Collier, The dual of a space with the Radon-Nikodym property, Pacific J. Math. 64 (1) (1976), 103-106.

[DGS-R95] G. Debs, G. Godefroy, and J. Saint Raymond, Topological properties of the set of norm-attaining linear functionals, Canad. J. Math. 47 (2), (1995), 318-329.

[DGHZ87] R. Deville, G. Godefroy, D. E. G Hare, and V. Zizler, Differentiability of convex functions and the convex point of continuity property, Israel J. Math. 59 (1987), 245-255.

[DGZ93] R. Deville, G. Godefroy, and V. Zizler, Smoothness and renormings in Banach spaces, Pitman Monographs 64, London, Logman, 1993. 
[Di75] J. Diestel, Geometry of Banach Spaces-Selected Topics, Lecture Notes in Mathematics 485, Springer 1975.

[Ede73] M. Edelstein, Concerning dentability, Pacific J. Math. 46 (1) (1973), 111-114.

[EdWh84] G. A. Edgar and R. H. Wheeler, Topological properties of Banach spaces, Pacific J. Math. 46 (1984), 317-350.

[FHHMZ11] M. Fabian, P. Habala, P. Hájek, V. Montesinos, and V. Zizler, Banach Space Theory. The Basis for Linear and Nonlinear Analysis, CMS Books in Mathematics, Springer-Verlag, 2011.

[Fa97] M. Fabian, Differentiability of convex functions and topology: Weak Asplund spaces, Wiley 1997.

[HHZ96] P. Hájek, P. Habala, and V. Zizler, Introduction to Banach spaces I, II, Matfyzpress (Charles University text), Prague 1996.

[HMVZ08] P. Hájek, V. Montesinos, J. Vanderwerff, and V. Zizler, Biorthogonal Systems in Banach spaces, CMS Books in Mathematics, Springer-Verlag, 2008.

[HMZ11] P. Hájek, V. Montesinos, and V. Zizler, Geometry and Gâteaux smoothness in separable Banach spaces, Oper. Matrices 6 (2012) (2), 201-232.

[Ka91] R. Kaufman, Topics on analytic sets, Fundamenta Math. 139 (1991), 215-229.

[Ka00] R. Kaufman, On smooth norms and analytic sets, Israel J. Math. 116 (2000), 21-27.

[Ku11] O. Kurka, Structure of the set of norm-attaining functionals on strictly convex spaces, Canad. Math. Bull. 54 (2), (2011), 302310 .

[MZZ15] V. Montesinos, P. Zizler, and V. Zizler, An Introduction to Modern Analysis, Springer, 2015.

[Ph93] R. R. Phelps, Convex Functions, Monotone Operators and Differentiability, 2nd. Ed., LNM 1364, Springer-Verlag, 1993.

[PrZa84] D. Preiss and L. Zajíček, Fréchet differentiability of convex functions in a Banach space with a separable dual, Proc. Amer. Math. Soc. 91 (1984), 202-204. 
Instituto de Matemática Pura y Aplicada, Universitat Politècnica de València, Camino de Vera, s/n. 46022 Valencia, Spain

E-mail address: anguisa2@mat.upv.es

Instituto de Matemática Pura y Aplicada, Universitat Politècnica de València, Camino de Vera, s/n. 46022 Valencia, Spain

E-mail address: vmontesinos@mat.upv.es

Department of Mathematics, Central Academic Building, University of Alberta, Edmonton T6G 2G1, Alberta, Canada

E-mail address: vasekzizler@gmail.com 